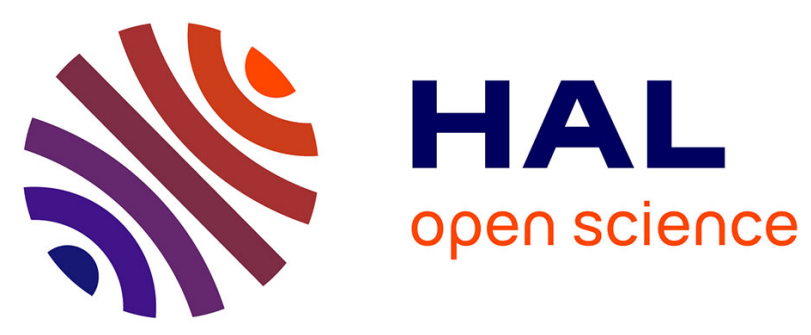

\title{
Designing an IIR Research Apparatus with Users with Severe Intellectual Disability
}

Filip Bircanin, Laurianne Sitbon, Benoit Favre, Margot Brereton

\section{To cite this version:}

Filip Bircanin, Laurianne Sitbon, Benoit Favre, Margot Brereton. Designing an IIR Research Apparatus with Users with Severe Intellectual Disability. ACM SIGIR Conference on Human Information Interaction and Retrieval (CHIIR), Mar 2020, Vancouver, Canada. pp.412-416, 10.1145/3343413.3378008. hal-02470797

\section{HAL Id: hal-02470797 \\ https://hal-amu.archives-ouvertes.fr/hal-02470797}

Submitted on 6 Nov 2020

HAL is a multi-disciplinary open access archive for the deposit and dissemination of scientific research documents, whether they are published or not. The documents may come from teaching and research institutions in France or abroad, or from public or private research centers.
L'archive ouverte pluridisciplinaire HAL, est destinée au dépôt et à la diffusion de documents scientifiques de niveau recherche, publiés ou non, émanant des établissements d'enseignement et de recherche français ou étrangers, des laboratoires publics ou privés. 


\section{Designing an IIR Research Apparatus with Users with Severe Intellectual Disability}

\author{
Filip Bircanin \\ f.bircanin@qut.edu.au \\ Queensland University of Technology \\ Brisbane, QLD, Australia \\ Benoit Favre \\ benoit.favre@lis-lab.fr \\ Aix Marseille Univ, Universite de Toulon, CNRS, LIS \\ Marseille, France
}

\author{
Laurianne Sitbon \\ 1.sitbon@qut.edu.au \\ Queensland University of Technology \\ Brisbane, QLD, Australia \\ Margot Brereton \\ m.brereton@qut.edu.au \\ Queensland University of Technology \\ Brisbane, QLD, Australia
}

\begin{abstract}
Traditional methods of engagement with pre-defined queries, verbal instruction and interviewing do not provide necessary means to address information-seeking behavior and visual browsing for participants with severe autism and intellectual disability. In this paper, we identify challenges and characteristics of providing effective methods to explore visual browsing and video recommender systems with one non-verbal participant with autism and intellectual disability. We contribute a case study and a reflection on a) how iterative design approaches that builds on special interests and strengths of one individual with disability can support experimental IIR research in becoming more inclusive, b) some of the ethical consideration that arise in the tensions between participation in the research and other interests and c) how flexible experimental and apparatus design can further allow participant's terms to prevail.
\end{abstract}

\section{CCS CONCEPTS}

- Human-centered computing $\rightarrow$ Human computer interaction (HCI); Accessibility; $\bullet$ Information systems $\rightarrow$ Users and interactive retrieval.

\section{KEYWORDS}

IIR experimentation, intellectual disability, browsing

ACM Reference Format:

Filip Bircanin, Laurianne Sitbon, Benoit Favre, and Margot Brereton. 2020. Designing an IIR Research Apparatus with Users with Severe Intellectual Disability. In 2020 Conference on Human Information Interaction and Retrieval (CHIIR '20), March 14-18, 2020, Vancouver, BC, Canada. ACM, New York, NY, USA, 5 pages. https://doi.org/10.1145/3343413.3378008

\section{INTRODUCTION AND RELATED WORK}

There is a scarcity of interactive information retrieval (IIR) research involving people with intellectual disability [10], particularly when it comes to exploring information access by people who are nonverbal. There has been research in designing interfaces and new

Publication rights licensed to ACM. ACM acknowledges that this contribution was authored or co-authored by an employee, contractor or affiliate of a national govern ment. As such, the Government retains a nonexclusive, royalty-free right to publish or reproduce this article, or to allow others to do so, for Government purposes only.

CHIIR '20, March 14-18, 2020, Vancouver, BC, Canada

(C) 2020 Copyright held by the owner/author(s). Publication rights licensed to ACM ACM ISBN 978-1-4503-6892-6/20/03 ..\$15.00

https://doi.org/10.1145/3343413.3378008 modes of interaction for non literate participants, such as children [3]. However, the focus is on media in very small datasets, which relates to information presentation rather than interaction or search. Furthermore, small children in these studies could understand instruction and verbally express themselves.

Following one participant's lead through his demonstrated browsing skills within the Youtube video repository interface, we set more specifically to explore how targeted browsing could support information seeking. Visual browsing is part of the broader field of visual information seeking [2], and generally comprises exploratory tasks (without a specific goal) and targeted search [7]. As previous work suggests [5], engaging people with cognitive disability in IIR research requires a reconsideration of our methodology, how we are setting search tasks and engaging participants in our experiments.

In this paper, we present a case study where we have effectively designed an IIR research apparatus that would allow us to collect and explore interactions of one non-verbal participant with severe autism and intellectual disability ${ }^{1}$ in a controlled experimental environment. Through reflection on this case study, we hope to unveil challenges and questions which arise when we seek to engage with participants with severe intellectual disability in IIR research.

Our approach to design a research apparatus used the methods of participatory design as they have been adapted and applied to be effective with people with intellectual disability: strength-based engagement [11, 12], iterative design [6], and reflection in action [11]. This approach is also inspired by Rapid Agile Iterative Design [9] and its adaptation as design after design when working with people with intellectual disability ([6]). It consists in deploying iterative prototypes and reflecting after short-term trials and observations. In addition to reflecting between iterative cycles, we also incorporated principles of reflection-in-action [11] to dynamically adapt the prototypes during trials, where possible and appropriate.

We present our observations, reflections and research tools in each iteration within the format of a case study. This case study mainly focuses on 1 non-verbal participant, Alex, with severe autism and intellectual disability. This case study was part of a broader project which is approved by the Queensland University of Technology ethics committee under ethical clearance 1400000673.

${ }^{1}$ Intellectual disability is the preferred terminology in Australia at the time of writing 


\section{CASE STUDY}

\subsection{Preliminary observations}

2.1.1 Context. The case study was conducted in a Learning and Lifestyle centre (community day centre) ran by the Endeavour Foundation, a disability service organisation supporting people with intellectual disability, in a large city in Australia. The centre offers daily assistance in educational and recreational activities for 30-35 adults with cognitive disabilities in the age range of 19-55. One of the researcher on the team, at the beginning of the study, had already engaged in long-term engagement in this particular community centre for over a year.

2.1.2 Observations. During our long-term engagement in the community centre, we noticed Alex, a young adult who enjoys his tablet and the YouTube App. Alex has complex communication difficulties - he cannot write, he cannot type and he cannot speak. He uses short utterances and vocalisations to express his satisfaction or dissatisfaction. He has been diagnosed as someone having severe autism and intellectual disability.

Alex has an aspiration towards one particular video of interest (Spirit, a short video animation). He watches this video in different languages, focusing only on particular visual or sound effects of the videos and spending hours on the YouTube app watching it.

We had been running a workshop with other participants about search on Youtube [4], and support workers had suggested to invite Alex to the workshops. While we were unsure what a suitable way of engaging Alex in the workshop might be, the support workers indicated that he would potentially benefit from simply sitting with us. During most workshops, Alex would stay in the room and sit at the back on his iPad. However, during our regular workshop activities and nearing the end of our tri-semester cycle of technology workshops, Alex came up to the screen as he was walking in, and spontaneously demonstrated his ability to retrieve his video of interest. As Alex is known to be non-verbal, he does not use the search function to reach his goal, but navigates through the recommendations. We had already observed him doing this fast and efficiently on his device. However, this was a different screen as well as a different device. As a result, recommendations came up differently than on his own device. We tried again on a different tablet with no prior history, and he was again able to very rapidly find a video with his favourite character.

After two months (and when we became closer to Alex), we have explored again if he would be able to find the video if the history of YouTube was completely erased. In one turn he was able to do that whereas in the other turn, just a few days later, he was no longer able to find the desired video. Since we have no control over the recommendation algorithm of Youtube, we assumed that something changed that may have set him off course.

2.1.3 Reflection. Alex had a known interest in Youtube and demonstrated ability to access content of his choice. The skill of browsing through recommendations to reach target content could be explored further into a broader scope of information access. However, we need to understand what strategies Alex employs to reach his goal (eg. the nature of his associative behaviour). Further to this, staff are interested to explore how this interest in Youtube and one particular video could lead to an application that could support Alex and expand upon his daily living skills through videos starting with the question: "can we introduce new content to him"?

Building a dedicated application would allow the research team to proceed forward with 2 methods: a) offering, logging and analysing the choices made by Alex when it comes to selecting the route to browse towards his target, and b) iteratively design an application that could expand the scope of videos accessed by Alex. A challenge that now arises with introducing a new application, is that, even if it allows to watch the same videos as the platform Alex is used to, it would derive from a habit of using Youtube built over many years. Additionally, he may not know that the content he favours could be available in the new platform. He may also not understand that we are asking him to retrieve it.

\subsection{First Iteration}

In the first iteration, we started building an application which supports the browsing capabilities of Alex, and builds on videos and recommendations from the Youtube platform.

2.2.1 Application. The application resembles the YouTube app while allowing us to explore Alex's behaviour. At the same time, it lets him have full control as all interactions are based on visuals. Starting from the design of the Youtube's app, we have removed all features that we hadn't seen Alex using so far (text, voice search, commenting, unnecessary symbols, advertisements, etc.).

It is built as a web application and makes use of the Youtube API in order to access thumbnails, videos and recommendations. It can run across platforms, in the browser. A major advantage of a web application is the flexibility to modify the application as necessary while a participant is using it, thus not interrupting a trial. The display of the application is composed of 50 thumbnails videos presented a scrollable vertical grid. On the left-hand side, the thumbnails are of videos recommended by the Youtube platform. On the right-hand side, the videos are selected from a random set of videos available on Youtube as well.

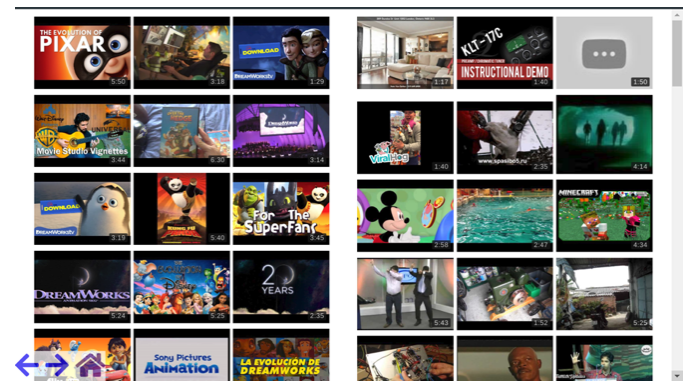

Figure 1: First iteration of the application, with only thumbnails. It shows a full screen image of the target video (thumbnail) and only the target video plays when it is found.

As browsing behaviours are typically exploratory rather than target driven, existing experimental frameworks mostly leave users free to operate, and analyse the journey as much as the end point. In our case, we were interested to understand how Alex is using associative browsing to reach a goal, and from there how we may support this behaviour to help him access new content. In addition 
to filming Alex during interactions, we setup a simple logging of the different videos he selects on the interface. We also tracked user interaction with a full screen recorder ${ }^{2}$ which we created for Android and records screen touches, screen content, and audio captured by the microphone as a video saved on the device. This kind of analysis tool was useful for tracking fine-grained user behaviour inside and outside the app.

As Alex hardly communicates with people in his surroundings, it is difficult to know if he understands what we want him to do with this interface. As a result, we have designed a visual task setting feature, in addition to basing the task on his known interest When the homepage of the application is loaded, a full-screen thumbnail of the Spirit horse video appears and then fades in the background. With this we hoped that Alex would understand our task instructions - looking for the desired video (spirit horse) within the database of shown videos. In addition to the thumbnails, the screen presents in permanent overlay (static on the screen when scrolling down occur) 3 buttons, which are intended as controls for the researcher during the trials. The arrows allow navigating back and forth through previous actions, and the home button restarts the application with the static image of the Spirit horse.

2.2.2 Context. Alex was using his iPad in the past to communicate via an Augmented Alternative Communication (AAC) device (Proloquo2Go (P2Go) [1]) manipulating pictograms and symbols on his device (e.g."I want food"). However, it had become difficult for parents and staff to keep Alex motivated as he was constantly switching from P2GO to YouTube and videos of interest. He had broken two iPads from frustration of waiting for videos to load on his iPad. Some staff members in the Day centre say that he is 'addicted to screens" and that he mainly enjoys the videos on YouTube. As Alex was socially isolated because of this apparent compulsion to using the tablet, at the time we introduced this first application, he was no longer allowed to use an iPad while 3 at the centre. An exception was made for our research trials, which in turn was the only opportunity for Alex to access a tablet and videos.

2.2.3 Observations. Upon seeing the image of the spirit horse Alex was excited to continue with the search for the video. However, after 5 minutes of using the interface, Alex was showing difficulties navigating the interface. As he was not able to find the video, he exited the interface, looking for the YouTube app on the iPad. As the YouTube app was previously deleted, he launched the browser to find the YouTube website. He then clicked on the URL box where the history of previous websites used was shown. In the list, on the fourth place the Youtube platform was listed without the YouTube symbol. We then learned that Alex can recognize the shape of the letters without having a help or prompt with the symbol. We knew that he knows how to recognize the YouTube symbol but we did not know that he can recognize the shape and the organizational structure of the letters.

Once Alex managed to open the YouTube app in his browser, he was again no longer able to retrieve his video on the Youtube platform through browsing. Hence, he went back to our platform, trying one more time to find the desired Spirit video. He had again

\footnotetext{
${ }^{2}$ Source code available at https://urldefense.com/v3/_https://github.com/ design-participation/android-screenrecorder_;!!NVzLfOphnbDXSw!SZj-

ZUqWuCwzpVsdpCShuFAe4ay159J2c6qz57BzWSeZ-4WPiydK2zL4cwDrxl194Oc\$
}

no success with finding the video. He thus started using the home button in the lower part of the interface screen that upon pressing would show the picture of the spirit horse fading in the background. He started repeatedly launching that feature (the spirit picture) content with by seeing the image of the spirit horse.

2.2.4 Reflection. One of the support workers suggested to maybe introduce categories of related videos, and simplifying the interaction by introducing on the right-hand side of the screen 4 different categories that might speed up the whole process of the searching for the spirit video. He suggested using Marvel, Pixar, Disney and Dreamworks as the Spirit animation belongs to one of these categories (Dreamworks). In addition, Alex knows that Youtube will immediately reward him.

\subsection{Second Iteration}

2.3.1 Application. In the second version of the application, after the first thumbnail is selected, the top part of the screen plays the video, while the bottom part provides a new set of images to choose from to continue browsing. The video player on the top of the screen allows interaction (watching and controlling videos) with any chosen videos of interest. This particular feature was inspired by allowing full autonomy and control over the selected videos (playing and pausing). It also allowed us to explore if Alex might engage with sound and visual effects of other related videos that are not the target video (Spirit video).

The grid of suggestions still comprises 50 images, which are thumbnails of videos. The left hand side is still comprised of recommendations from Youtube, however the right-hand side of the interface now has a random set of videos where $25 \%$ are from the category of Disney, Dreamworks, Marvel and Pixar and $75 \%$ of a random set of 100,000 YouTube videos. This way, we have increased the chance for Alex to be able to find the video. In addition to changes to the display, we also deployed the application using the "kiosk" mode ${ }^{3}$, which prevents users (who are not administrators) from navigating outside the web application.

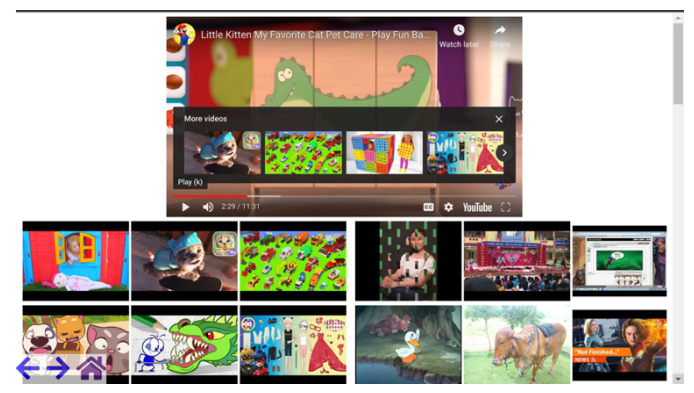

Figure 2: Second iteration of the application, with video player at the top and thumbnails on the bottom half of the screen. It also shows a full screen image of the target video.

\footnotetext{
3available at https://urldefense.com/v3/_https://github.com/ design-participation/android-kioskweb_,;!NVzLfOphnbDXSw!SZj-

ZUqWuCwzpVsdpCShuFAe4ay159J2c6qz57BzWSeZ-4WPiydK2zL4cwDrvU2zEpA\$
} 
2.3.2 Context. This second iteration was trialed over a day. When we arrived at the centre, we presented the new version of the application to Alex and he trialed it for one session. Then he went sleeping. We waited 2 hours for him to wake up, and then made sure he was ready to have another go at trialling the application.

2.3.3 Observations. With the 4 categories (Disney, marvel etc.) on the right-hand side, Alex was able to find a Spirit horse video in 5 clicks on the first trial. He then kept looking for more with 2 more interactions, demonstrating his understanding that the recommender system would take him to one he preferred. Then Alex had a rest, and we reflected on the spot that he did not seem this time to have needed the motivation of the preview video at the beginning. In order to assess this, we removed it from the application.

We then restarted the application. At that point, Alex could no longer find the spirit video. After one minute, he tried to exit the application. However, this would not work as the application was running in kiosk mode. He then resumed browsing with the application for over 20 minutes. He was happy with content that was similar (other cartoons). He looped through those videos by using the arrows to go back and forth through the videos in his history of selected and watched videos.

In both instances, Alex used the video player on each screen to skim through the videos as a more informed preview than the thumbnails themselves. He very quickly decided to watch or skip the video using this feature. He also liked to interact with the player regardless of its relevance for browsing.

2.3.4 Reflection. As in the first iterative cycle, we have coincidentally learned that Alex knows more than we expected. Arrows and the home button at the bottom of the screen were designed for research team, but Alex has transferred his knowledge of the YouTube app to the new platform (new interface). By constraining Alex to stay within the bounds of our experimental application as long as he wanted to continue with the activity, we were able to observe a new behaviour of browsing without a target, and enjoying a new set of videos.

\section{DISCUSSION}

\subsection{Individuality}

Understanding the interests of Alex prior to experimenting with the application has played a major role in both designing the application itself but also the experimental setup. It allowed us to build in the app his attraction for the cartoon video in the search scenario. It also provided a way to provide both instructions on the task we were hoping him to perform, as well as motivation for him to proceed.

Recognising participants' interests and abilities has been widely recognised as a way to build applications that are engaging and effective $[11,12]$. However, while approaches in interaction design would encourage freedom of participants to demonstrate what they are most wanting to engage with, they often build on these interests and abilities to design for social participation or communication.

\subsection{Ethical Considerations}

In order to engage Alex in our research tasks, we built on his interests but we also effectively employed other persuasive strategies: locking the device in kiosk mode, (unintentionally) making the device the only way to access videos while attending the centre. Researchers can perhaps legitimately constrain the use of the tools they provide within the scope of the research. However, this raises questions on what is to become of the tool we started to design, given that in the comparatively short term of the study, Alex has demonstrated that his preference would remain for the platform he is most familiar with.

Short term impulses and turning to habits may not necessarily reflect long-terms values, and it is possible that after building a habit of using the tool we have designed with Alex, he would demonstrate a preference for the tool. However, it is important here to reflect on how restricting Alex's choice and control, in other words, autonomy, is meant to serve a broader purpose of increasing his independence. Design considerations on the tool as a learning platform to encourage him to learn new skills are beyond the scope of this paper. Yet it provides a good opportunity to reflect on whose values our investigation is echoing, as indeed "persuasive technology calls into question the value of autonomy " [8].

\subsection{Engagement in the Moment}

As this work was part of a longer term engagement, we were able to take the opportunity of an established mutual trust with Alex. It was also this long-term engagement that revealed a design space to explore. In addition, to a long-term relationship with Alex, it was important to understand how to time the trial in his terms. During the second iteration, it meant reading signs of tiredness and allowing him take a break before resuming his engagement instead of interrupting the trial altogether. We knew that he may not stay focused for long periods of time. It was therefore important to be reactive and able to flexibly adapt the application to respond to a question that had arisen from reflection in the moment.

\section{CONCLUSION}

We have presented and reflected on the iterative design of a application that can help us understand and log the browsing patterns of a participant on a video platform. Traditional experimental setup, with pre-defined queries, verbal instructions, and standard environment would have failed to engage with this particular participant. However, it may suit other participants with intellectual disability. We have provided insights into how personalisation and flexibility of the experimental procedure itself, as well as deep engagement with the participant, were important. We have also opened up a reflection on the role of persuasion to allow time for participants to appreciate the benefits a new technology may provide them with.

In future work, we will continue to collect and explore Alex's browsing skills and consider how the logs can support an adaptive system of recommendations. We will additionally create a feedback loop with family and support workers by presenting results and inviting them to participate in the co-design of new iterations of the application.

\section{ACKNOWLEDGMENTS}

This research has been supported by the Australian Research Council under grant LP160100800, with the joint support of the Endeavour Foundation. We wish to extend special thanks our participant for his time and valuable insights. 


\section{REFERENCES}

[1] [n.d.]. Proloquo2Go - AAC app with symbols - AssistiveWare. https://www. assistiveware.com/products/proloquo2go

[2] Dan Albertson. 2015. Visual information seeking. Fournal of the Association for Information Science and Technology 66, 6 (2015), 1091-1105. https://doi.org/10 1002/asi.23244

[3] Leif Azzopardi, Douglas Dowie, and Kelly Ann Marshall. 2012. YooSee: A Video Browsing Application for Young Children. In Proceedings of the 35th International ACM SIGIR Conference on Research and Development in Information Retrieval (SIGIR '12). ACM, New York, NY, USA, 1017-1017. https://doi.org/10.1145/ 2348283.2348442 event-place: Portland, Oregon, USA.

[4] Andrew A. Bayor, Laurianne Sitbon, Bernd Ploderer, Filip Bircanin, and Margot Brereton. 2019. "TechShops" Engaging Young Adults with Intellectual Disability in Exploratory Design Research. In Extended Abstracts of the 2019 CHI Conference on Human Factors in Computing Systems (CHI EA '19). ACM, New York, NY, USA CS31:1-CS31:8. https://doi.org/10.1145/3290607.3299056 event-place: Glasgow, Scotland Uk.

[5] Gerd Berget and Andrew MacFarlane. 2019. Experimental Methods in IIR: The Tension Between Rigour and Ethics in Studies Involving Users with Dyslexia. In Proceedings of the 2019 Conference on Human Information Interaction and Retrieval (CHIIR '19). ACM, New York, NY, USA, 93-101. https://doi.org/10.1145/3295750. 3298939 event-place: Glasgow, Scotland UK.

[6] Margot Brereton, Laurianne Sitbon, Muhammad Haziq Lim Abdullah, Mark Vanderberg, and Stewart Koplick. 2015. Design after design to bridge between people living with cognitive or sensory impairments, their friends and proxies. CoDesign 11, 1 (Jan. 2015), 4-20. https://doi.org/10.1080/15710882.2015.1009471 [7] Sally Jo Cunningham and David M. Nichols. 2008. How people find videos. In 7CDL '08. ACM, 201-210. https://doi.org/10.1145/1378889.1378924

[8] Batya Friedman and David Hendry. 2019. Value sensitive design: shaping technology with moral imagination. The MIT Press, Cambridge, Massachusetts.

[9] Clint Heyer and Margot Brereton. 2010. Design from the Everyday: Continuously Evolving, Embedded Exploratory Prototypes. In Proceedings of the 8th ACM Conference on Designing Interactive Systems (DIS '10). ACM, New York, NY, USA, 282-291. https://doi.org/10.1145/1858171.1858222 event-place: Aarhus, Denmark.

[10] L. Sitbon, A. Bayor, F. Bircanin, S. Koplick, and M. Brereton. 2018. An exploration of how people with intellectual disability engage with online information retrieval. Conference on Human Factors in Computing Systems - Proceedings 2018-April (2018).

[11] Cara Wilson, Margot Brereton, Bernd Ploderer, and Laurianne Sitbon. 2019. CoDesign Beyond Words: 'Moments of Interaction' with Minimally-Verbal Children on the Autism Spectrum. In Proceedings of the 2019 CHI Conference on Human Factors in Computing Systems (CHI '19). ACM, New York, NY, USA, 21:1-21:15. https://doi.org/10.1145/3290605.3300251 event-place: Glasgow, Scotland Uk.

[12] Jacob O. Wobbrock, Shaun K. Kane, Krzysztof Z. Gajos, Susumu Harada, and Jon Froehlich. 2011. Ability-Based Design: Concept, Principles and Examples. ACM Trans. Access. Comput. 3, 3 (April 2011), 9:1-9:27. https://doi.org/10.1145/ 1952383.1952384 